\title{
Carnets
}

Revue électronique d'études françaises de l'APEF

Deuxième série - $16 \mid 2019$

Le Récit inachevé: études sur Mai 68

\section{Mai est un roman}

\section{Luís Carlos Pimenta Gonçalves}

\section{(2) OpenEdition}

\section{Journals}

\section{Édition électronique}

URL : http://journals.openedition.org/carnets/9650

DOI : 10.4000/carnets. 9650

ISSN : 1646-7698

Éditeur

APEF

\section{Référence électronique}

Luís Carlos Pimenta Gonçalves, « Mai est un roman », Carnets [En ligne], Deuxième série - 16 | 2019, mis en ligne le 31 mai 2019, consulté le 12 novembre 2019. URL : http://journals.openedition.org/ carnets/9650; DOI : 10.4000/carnets.9650

Ce document a été généré automatiquement le 12 novembre 2019.

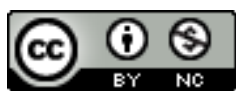

Carnets est mis à disposition selon les termes de la licence Creative Commons - Atribution - Pas d'utilisation commerciale 4.0 International. 


\title{
Mai est un roman
}

\author{
Luís Carlos Pimenta Gonçalves
}

1 Le roman de mai reste à écrire tant les passions qu'il a suscitées jusqu'à aujourd'hui perdurent. Événement célébré par les uns et honni par les autres. Ainsi, un ancien président de la République française ne promettait-il pas en 2007 d'en finir avec mai 68 ? En 2018, la mémoire collective semble intégrer de façon plus consensuelle les transformations sociales de l'après-mai comme le révèle l'opinion positive que s'en fait une très large majorité de sondés pour Le Nouveau Magazine littéraire en janvier dernier. En revanche, en parler à chaud, dès l'été 1968, s'en inspirer et l'inclure dans un texte littéraire, peu s'y sont risqués. Curieusement, le premier texte littéraire, fin 1968, est signé d'un politologue, Frédéric Bon, et d'un journaliste, Michel-Antoine Burnier, aidés de la complicité du dessinateur Wolinsky qui l'illustre de "graffitis». L'ouvrage s'intitule, Si mai avait gagné, sorte de récit de politique-fiction, décrit par le sous-titre comme une Facétie politique, reconstituant la victoire de la gauche en 1968 à partir de textes fossiles retrouvés au xxII ${ }^{\mathrm{e}}$ siècle. Revanche par l'humour, bien dans l'esprit de 68, de la défaite politique de la gauche dans la rue et dans les urnes cette année-là. En fait, beaucoup d'intellectuels et d'écrivains n'étaient pas loin de penser comme Marguerite Duras qui a déclaré quelques années plus tard: "On n’a pas eu le temps de vivre un événement aussi considérable que mai 1968 que, déjà, l'homme parle, passe à l'épilogue théorique et casse le silence ${ }^{1}{ }^{1}$ En parler à froid peut transformer l'exercice en un roman historique, genre habituellement utilisé pour décrire des événements éloignés dans le temps qui permettent toutes sortes de libertés avec les événements. Mai 68 qui a libéré la parole, mis à mal les codes anciens, l'autorité sous toutes ses formes, réclamé "L'imagination au pouvoir » et la "poésie dans la rue ", peut-il s'écrire sur le mode traditionnel du roman réaliste "à la Balzac ", décrié par les surréalistes, puis par les existentialistes et finalement par les auteurs étiquetés sous le signe du Nouveau roman? Question délicate et à laquelle ont répondu de manière diverse les romanciers qui ont abordé mai 68, même de façon latérale. Patrick Combes, auteur de Mai 68, Les écrivains, La littérature, publié en 2008 fait le constat suivant :

Mai n'a pas son Éducation sentimentale, où, comme pour 1848, les éléments politiques, sociaux, humains, seraient refondus, transformés, pour aboutir à une recréation - où l'expérience, l'enjeu historique seraient intégrés dans un roman de 
la condition humaine. Et la trouvera-t-il quand on considère les décennies qui nous en séparent? (Combes, $2008: 215-216$ ).

Il est difficile d'y répondre, tant les circonstances historiques du Printemps des Peuples et mai 68 sont différents. Il est vrai que le projet flaubertien était ambitieux qui, encore à l'état de projet, envisageait, selon les termes rapportés par les frères Goncourt : un " roman moderne » parlant à la fois du «mouvement de 1830 », de la "physionomie de 1840 , et 1848 , et l'Empire ", et qui s'intéresserait de surcroît aux "amours d'une Parisienne ».

3 En somme, résume Flaubert lors de cette conversation rapportée le 11 février $1863:$ « Je veux faire tenir l'Océan dans une carafe $»^{2}$. Au cours de cette communication et paraphrasant la formule utilisée par Flaubert, nous observerons comment l'océan de mai est entré, tout entier ou non, dans trois carafes distinctes: celle de Robert Merle dans Derrière la vitre, celle de Raymond Jean dans Les Deux Printemps et finalement celle de Pascal Lainé dans L'Irrévolution.

4 Il faudrait chercher une première réponse sur la capacité qu'ont eu ces trois auteurs à faire le grand roman de synthèse de 68 dans l'accueil et la postérité de ces trois œuvres. Il est assez symptomatique que deux de ces romans n'ont pas eu de réédition récente. Celui de Raymond Jean, Les Deux Printemps, édité originellement au Seuil en 1971, est repris en poche dans la collection 10/18 complété d'une introduction en 1978, à l'occasion du dixième anniversaire de mai 68. Quant à celui de Pascal Lainé, L'Irrévolution, édité en 1971 par Gallimard, dans la collection Le Chemin dirigée par George Lambrichs, il ne connaîtra pas de réédition. Meilleure postérité aura celui de Robert Merle, Derrière la vitre, édité tout d'abord par Gallimard en 1970, puis repris dans la collection Folio en 1974. Il est également édité par Tallandier, en 1971, suivi de "Fenêtre ouverte sur Robert Merle» propos recueillis par Daniel Lasagne, et finalement par Le Livre Club Diderot, en 1977, avec une préface de Raymond Jean, dans un volume intitulé Cuvres³. Derrière la vitre sera également adapté au théâtre en 1992 par Sylvie Gravagna sous le titre Nanterre-la-folie dont la première aura lieu justement dans un amphi de l'Université de Nanterre. Le relatif succès de ce roman est sans doute autant dû à ses qualités intrinsèques comme œuvre littéraire et document qu'au fait que l'écrivain avait déjà conquis un vaste public bien avant que ses romans historiques se passant au temps des guerres de religion en France ne le transforment en écrivain populaire. Robert Merle en adoptant un style qui évite les innovations formelles, surtout celles dérivées de l'existentialisme, du nouveau roman, voire de l'OULIPO, a su ainsi conquérir un lecteur sensible à ses thèmes philosophiques, politiques et sociaux mais qui est peu sensible aux mutations esthétiques du roman.

Quant à la reconnaissance immédiate des romans de notre corpus, un seul de ces trois ouvrages, L'Irrévolution, obtiendra un prix littéraire prestigieux à l'automne 1971, le Prix Médicis, qui, tels les autres ouvrages primés la même année, vendra 100000 exemplaires.

6 Les trois romans réunis pour notre exposé balisent en quelque sorte l'imaginaire des événements de 68. Tout d'abord, celui de Merle, Derrière la vitre, s'intéresse à la journée du 22 mars 1968, à Nanterre, avec quelques analepses qui évoquent des événements antérieurs dans cette même faculté. Unité de temps et de lieu, quoi de plus classique! Raymond Jean avec Les Deux Printemps nous propose de revisiter la nuit des barricades à Paris du 10 mai 68 dans la partie centrale du roman. L'unité de temps et de lieu ne se réalise que dans cette deuxième partie du livre puisque les deux autres se passent à 
Prague en 1969. Pascal Lainé aborde, lui, la première rentrée d'un jeune professeur de philosophie après les événements de 68 où seules de rapides allusions permettent de contextualiser le texte sur le plan politique et de l'histoire immédiate.

$7 \quad$ Le péritexte de Derrière la vitre inclut une présentation du roman sur deux pages (7 et 8) et surtout une préface de l'auteur non datée. On y apprend que le projet du livre naît en novembre 1967 lorsque Robert Merle demande à ses étudiants de la Faculté de Nanterre de l'«aider à mieux les connaître " lors d'entretiens. Le résultat est surprenant de franchise et étonne même l'enseignant. Il bénéficie de cette façon d'une sorte de mise à jour « au niveau du moins des idées et de langue » (Merle, 2008 : 10), écrit-il. Alors que l'idée initiale était simplement de décrire la vie quotidienne des étudiants à Nanterre, les événements de 1968 bouleversent cette intention première et il choisit de traiter la journée du 22 mars qu'il suit dans le roman heure par heure.

8 Après un premier chapitre de géographie physique et humaine, dans le droit fil du roman balzacien, le deuxième chapitre se déroule le « 22 mars, 6 heures du matin » et le dernier à « 23 heures trente». Au lieu de donner à cette journée une valeur exceptionnelle, ce qu'elle fut pour les 142 étudiants qui prirent directement part à l'occupation de la tour administrative et de la salle du Conseil des professeurs, elle demeure pour le plus grand nombre des 12000 étudiants une journée comme les autres. Ainsi, selon Merle : «Pour le romancier, pourtant, qui cherche à retrouver la vérité du moment sous les fastes de l'Histoire, le 22 mars appartient à la quotidienneté de Nanterre et ne doit pas en être détaché ». Dans cette même préface, l'auteur qui fait usage de personnages réels qu'il cite : "le doyen Grappin, l'assesseur Beaujeu, le secrétaire général Rivière et, du côté étudiant, Cohn-Bendit, Duteil, Tarnero, Xavier Langlade », crée également de toutes pièces des personnages fictionnels. Refusant que son texte ne soit considéré comme un roman à clefs cachant sous des noms d'emprunt des noms bien réels comme le firent en leur temps Simone de Beauvoir avec Les Mandarins ou, plus tard, Julia Kristeva avec Les Samouraïs, Merle dissocie son travail d'écrivain de celui de " ces peintres du Moyen Âge qui, dans un tableau grouillant d'hommes et de femmes, s'amusaient à dessiner leur voisin boulanger dans un coin de la toile ». Ainsi, s'il lui est arrivé, filant la métaphore de l'art du peintre, de «croquer d'après nature " ce n'est que pour mieux rendre crédibles ses personnages imaginaires en faisant usage des procédés du montage et du collage : «les personnages importants sont des portraits composites créés à partir de modèles divers empruntés à ma vie de professeur, et pas nécessairement nanterriens ». Pour rappel, l'auteur qui a déjà une longue carrière derrière lui a enseigné également dans les facultés des Lettres de Rennes, Toulouse, Caen, Alger et Rouen. Afin de mieux rendre la vie d'une multitude de personnages, réels ou inventés, où le sentiment de solitude et d'incommunicabilité, souvent évoqué dans les confidences d'étudiants, est renforcé par le fait que de nombreux épisodes se déroulent dans le confinement des chambres de la résidence universitaire, l'auteur a utilisé le simultanéisme (Merle, 2008: 11). Ce procédé littéraire en vogue, selon l'auteur, dans les années trente du vingtième siècle, songeant sans doute au cycle Les Hommes de bonne volonté de Jules Romains, a été délaissé par la suite. Au passage, Merle dans sa préface en profite pour épingler la critique qui avait mal accueilli, en 1962, un de ses précédents romans, L'île, usant du même artifice littéraire jugé déjà alors "démodé ». Ce faisant, il fait preuve dans sa préface d'un misonéisme sur le plan esthétique : "Je me méfie des systèmes qu'un artiste croit bon de se fabriquer. Je note que c'est toujours par où il se croit le plus neuf qu'il vieillit le plus vite. Si son œuvre survit, c'est pour d'autres mérites » (ibid.: 12). La conclusion de la préface indique le 
caractère partagé, ambigu, voire un certain malaise de Merle vis-à-vis des situations et des événements qu'il décrit dans son roman. Le roman révèle les événements qui

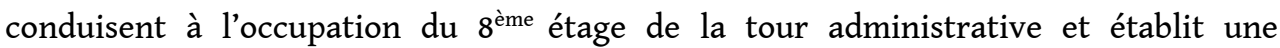
multitude de portraits d'enseignants, d'étudiants et de travailleurs immigrés algériens. Il met en scène les échanges et les relations qui se nouent. Ces rencontres rendues possibles grâce à la proximité géographique sont parfois hasardeuses. Ainsi, David, jeune étudiant et militant maoïste, ayant sa chambre à la résidence universitaire, et Abdelaziz, jeune ouvrier algérien habitant le bidonville de la Garenne tout proche et travaillant à la construction de la faculté, se lient d'amitié. Pourtant, la première rencontre est d'abord un échec, ils se voient symboliquement à travers une ouverture de la résidence le 22 mars vers midi :

« David regarda à nouveau les ouvriers répandre le goudron sur la terrasse. Celle-ci était à peu près à un mètre de son étage, il n'était séparé d'eux que par une vitre, mais de son côté à lui, tout était tiède et propre. C'était un monde où personne ne suait à manipuler les choses » (ibid.:121-122) et un peu plus loin le sentiment du personnage est sans équivoque « David se sentit coupable et malheureux» (ibid. 129).

Cette rencontre improbable entre deux jeunes de milieux et d'origines si différents est tout d'abord rendu difficile par une barrière et un obstacle - une vitre non seulement les sépare, d'où le titre, mais également un chantier. Pour dépasser cette distance sociale et physique, David lance une cigarette qui s'écrase malencontreusement dans le goudron: "Il y eut un silence. Ils regardaient la cigarette souillée, noircie, perdue. C'était un échec. Ils se sentaient atteints par cet échec. » (ibid.).

Les personnages des fictions de notre corpus sont, dans leur grande majorité, issus de la bourgeoisie, effet de réel oblige, la réalité sociologique étant ce qu'elle est à l'époque. Ainsi, rares seront les figures de l'étudiant contestataire fils d'ouvrier. Merle crée Bourrelier, le patronyme est déjà en soi la traduction d'une lignée artisane et manuelle, qu'il décrit physiquement de façon archétypale et stéréotypée: "Au bout de ses manches trop courtes, sortaient de larges mains prolétariennes aux ongles carrés et sincères ». Bien différent est David Schultz, fils de chirurgien, et qui ne détonne pas avec les autres personnages. Étudiant engagé, il anticipe ce que pourrait l'expérience ouvrière pour un jeune intellectuel :

Et les dominés, là-bas, de l'autre côté de la vitre, courbés en deux dans le froid, le vent aigre, la pluie, les muscles tiraillés par l'effort comme des bêtes de somme, ils n'avaient jamais pas plus de chance de s'introduire jamais dans ce monde-ci que moi dans le leur. Oh, je sais, je pourrais moi aussi me faire embaucher dans une usine, Simone Weil ou l'imitation de Jésus Christ. Mais je ne serais jamais un ouvrier, je vivrais ma pseudo condition ouvrière comme un intellectuel qui peut $\mathrm{y}$ mettre fin à tout moment, au nom, précisément, du capital accumulé des ses idées, de ses diplômes, de son savoir-faire technologique (ibid. :122)

11 Le roman de Raymond Jean, Les Deux Printemps, est construit de façon fragmentaire. L'épitexte de l'édition de 1978 de ce roman pour la collection 10-18 comporte une introduction de l'auteur (p. v-xxII) ainsi qu'en fin d'ouvrage une liste de « Documents utilisés comme éléments de montage " (Jean, 1978: 254). Dans l'introduction de l'édition au format poche, l'auteur avoue sa déception face au mauvais accueil du roman quelques années auparavant. À l'opposé du roman de Merle qui dénonce dans sa préface des effets de mode et l'esthétique du roman des années 60, Raymond Jean est proche par son écriture du nouveau roman et de ses recherches formelles. Il intègre des textes provenant de la presse écrite, des discours politiques, des déclarations, des 
inscriptions murales et des reportages radiophoniques. Ce principe de montage qui n'a pourtant rien d'innovateur déjà à l'époque a été mal perçu par une partie de la critique. Ce que rappelle l'auteur dans son introduction:

En fait, les Deux Printemps n'ont pas eu de chance. Comme romancier, j'avais eu le tort d'y faire quelques recherches formelles qui faisaient dire à Pierre-Henri Simon (dans le Monde du 16 octobre 1971) que je serais bien avisé dans l'avenir de "préférer le stylo aux ciseaux et au pot de colle comme instrument de mon art » (ibid. : v).

12 Le jugement du critique et académicien, responsable alors de l'influent feuilleton littéraire du Monde, était sans appel et a, sans doute, nui à la diffusion de l'œuvre dont s'afflige l'auteur. Le tort et conséquemment l'insuccès du roman, selon Raymond Jean, tiennent également à la nature politique du texte et à sa position assez incertaine sur les événements de Prague qui fâche ses camarades du PCF et le rédacteur du journal L'Humanité, André Stil, qui critiquait alors «l'instabilité politique» du romancier. D'autant plus navrant que Raymond Jean, après avoir été un compagnon de route depuis la Libération, venait d'adhérer au Parti communiste. Il est vrai que le narrateur des Deux Printemps, professeur d'histoire à l'université, vit un double désenchantement politique pendant l'été 1968. La première et la troisième partie narrent le retour du narrateur personnage à Prague, qu'il a visité autrefois. La deuxième partie qui occupe une position centrale dans le roman (p.95 - 184) intitulée « La nuit du 10 au 11 mai 1968, en France » est construite comme une longue analepse narrative. Le narrateur étant un universitaire âgé d'une quarantaine d'années, la parenté entre le personnage et l'auteur s'impose. Lors d'une émission d'Apostrophes, du 19 janvier 1979, Bernard Pivot, comme il en était coutumier, affirme d'un ton goguenard à l'adresse de Raymond Jean : «Il est très proche de vous, le professeur », son invité l'interrompt aussitôt : «Ce n'est pas moi !» Ce que confirme Raymond Jean dans son introduction : «Je précise pourtant à ce sujet qu'il n'y a aucune identification à faire entre le narrateur de Deux Printemps et moi » (p. viII). L'argument qu'il utilise semble cependant bien léger :

Certes il parle à la première personne, mais, dès le début du livre, il dit : " Je suis historien. Je prépare en ce moment un livre sur la Commune, parce que nous approchons du centenaire et qu'une collection m'a fait une proposition que je n'ai pas refusée » (ibid. : VIII)

13 Les Deux printemps, au-delà de la simple évocation du Printemps de Prague et de celui de Paris, envisage le caractère double des deux événements. Les événements de Prague et la réforme du régime de l'intérieur, qui commence bien avant 68 , sont perçus bien différemment dans le roman par ceux qui ont pris fait et cause pour cette ouverture, comme l'étudiante pragoise Zdenka qui a séjourné à Paris en 68 , et ceux qui s'y sont farouchement opposés, se réjouissant de l'intervention des forces du Pacte de Varsovie, comme le professeur Skopocek qui prévient le narrateur dès son arrivée à Prague :

N'écoutez surtout pas ce qu'on vous raconte, a-t-il dit dans son français rocailleux, mais très correct, ici les gens sont devenus fous. Dans ma propre famille, ma femme est folle. Depuis que les camarades soviétiques sont venus à notre aide, elle ne couche plus avec moi (p.13).

Parallèlement à cette perception antinomique des événements pragois, nous avons celle de mai 68 à Paris, tout particulièrement la nuit des barricades du 10 mai. Le narrateur et deux couples d'étudiants, réunis dans le petit appartement de l'un d'eux, suivent les événements racontés à la radio par un reporter de France Inter, puis sur une radio périphérique, Europe № 1 . Un appel de Cohn-Bendit entendu à la radio suscite 
chez deux des personnages présents dans la pièce des réflexions opposées. Alors que Raphaël, présenté antérieurement au narrateur comme "étant au P.C» (ibid.: 108), réagit violemment, accusant Cohn-Bendit d'être un "agent provocateur ", Clément, étudiant proche de l'extrême gauche, le défend, provoquant une altercation entre eux. Puis, au détour d'une page surgit un autre printemps, celui de la Commune de Paris, un siècle plus tôt, convoqué par la seule force expressive d'un substantif: "Le mot de barricade m'a plongé dans une sorte de bizarre rêverie et je me suis mis à penser à mon projet de livre sur la Commune» (ibid.: 112). Le narrateur se souvient alors d'une gravure d'après Robida d'hommes entassant des pavés « quai de l'Hôtel-de-Ville peutêtre, en mars 71, un vrai rêve d'historien » (ibid.), précise-t-il.

Tel Raymond Jean qui refusait dans son introduction toute équivalence entre lui et le personnage central des Deux Printemps, Pascal Lainé prévient son lecteur dès la première page en un paragraphe se détachant de l'incipit :

L'auteur du récit affirme que les événements relatés ici, les lieux et les personnages décrits sont imaginaires, et que toute ressemblance avec des faits et des lieux réels, ou avec des personnes existant ou ayant existé, à commencer par celle de l'auteur lui-même, serait pure coïncidence. (Lainé, 1971 : 7)

Pourtant, tel son personnage, Pascal Lainé a été pendant deux années professeur dans un lycée technique et puise dans ses souvenirs matière à brosser portraits d'enseignants et d'élèves. Le roman narre l'histoire d'un jeune agrégé de vingt-six ans, affecté dans une ville imaginaire du Nord, Sottenville ${ }^{4}$ (p.12). Comme le titre le suggère, le roman ne traite pas d'une révolution qui aurait eu lieu en mai 68 , mais d'une "irrévolution ", le préfixe privatif forgeant le néologisme indiquant un contraire, une absence, une négation comme dans "irrépressible» ou «irrésolution ». Ce dernier exemple de substantif pourrait être d'ailleurs mis au compte du personnage de ce roman de Pascal Lainé. Il est véritablement irrésolu quant à son destin, s'étant longtemps laissé guider par sa mère, devenu enseignant de philosophie un peu malgré lui. À la fin du récit, le narrateur semble néanmoins vouloir échapper à un destin tout tracé quand, après un échec professionnel et une mésentente avec le directeur de son lycée, il décide de ne pas aller en cours et de rentrer à Paris. Grâce à de rares analepses au cours du récit, on découvre qu'il a davantage été témoin que participant des événements de mai 68. Incroyant en religion comme en politique et l'équivalence est toute faite au détour d'une phrase, il découvre cependant une foi momentanée en se mêlant à une manifestation :

Mai ! Que c'est déjà loin le joli mois de mai, et ses nuits en noir et blanc, sa lanterne magique! J'avais cru, pour la première fois. J'avais trouvé quelque chose qui ressemblait à la grâce au coin du boulevard Saint-Michel et la rue Soufflot (Lainé : 1971 :34-35).

Les événements de mai ont juste effleuré la prospère et paisible petite ville du nord et les revendications semblent bien dérisoires. Le directeur du lycée technique a réussi auprès du rectorat à obtenir des crédits supplémentaires en profitant d'un climat propice aux revendications. Le résultat est tristement ironique : un distributeur de boissons chaudes au lieu des deux initialement prévus. À chaque instant de ce récit, on ressent le total déphasage entre le narrateur et les autres personnages, sottenvillais ou parisiens. Il est comme les étudiants de Merle "derrière la vitre ", ici les travailleurs algériens sont simplement remplacés par des fils d'ouvriers, d'employés ou de petits commerçants. Mais il est également éloigné de la bourgeoisie à laquelle il appartient par sa famille jusqu'à ressentir un sentiment complexe de haine qui est en même temps 
une détestation de soi. Ce n'est que vers la fin du roman que l'on découvre le sens du titre et ce qui en fait un roman d'une génération écrit à la suite des événements de mai :

C'est cela mon mal; et peut-être, comme on dit, le «mal du siècle»; c'est l'irrévolution: c'est le mouvement contradictoire d'une inquiétude et d'une critique si profondes, si totales peut-être, qu'elles-mêmes n'échappent pas à leur propre acide, et qu'elles se dissolvent dans leur réflexion sur elles-mêmes, qu'elles s'effacent (ibid. :142)

Ce «mal du siècle » n'affecte pas seulement le narrateur ou quelques individus isolés, mais s'étend à une génération entière d'étudiants :

Je ne suis pas le seul de mon espèce. L'irrévolution, nous avons été tous à la vivre ainsi, en mai 68. Nous avons fait l'irrévolution, non la révolution, parce que nul de ceux qui devaient la faire, la révolution, parce qu'aucun de nous n'espérait y trouver vraiment son assouvissement. (ibid. : 143)

Par le biais de ces trois œuvres, nous avons essayé de rendre compte du roman de mai tel qu'il s'exprime et se conçoit au début des années 70. Aucun de ces romanciers ne s'est essayé à faire œuvre d'historien, Raymond Jean le récuse explicitement malgré la multitude de sources authentiques qui parsèment Les Deux printemps, tout au plus le genre de l'essai est perceptible au détour de nombreuses pages des trois auteurs. Aucun d'eux n'a eu l'ambition de circonscrire ce qu'a été 68 car mai est un roman immense qui reste à écrire.

\section{BIBLIOGRAPHIE}

COMBES, P. (2008). Mai 68, les écrivains, la littérature. Paris, L'Harmattan.

COHN-BENDIT, D. (1988). 1968 : A Revolução que tanto amámos! Lisboa, Publicações Dom Quixote, Lda.

COHN-BENDIT, D. (2008). Forget 68. La Tour d'Aigues, Éditions de l'Aube.

FLAUBERT, G. (2008). Correspondance, tome III. Paris : Gallimard, « Bibliothèque de la Pléiade ».

JEAN, R. (1978). Les Deux Printemps. Paris : Éditions du Seuil, « 10/18».

JOFFRIN, L. (1998). Mai 68. Histoire des Événements. Paris : Seuil, « Points ».

LAINÉ, P. (1971). L'Irrévolution. Paris : Gallimard.

LE GOFF, J.-P. (2006). Mai 68, l'héritage impossible. Paris : Éditions la Découvert, « Poche ».

LAURENTIN, E. (2008). « Le transistor à l'écoute de la rue ». In ARTIÈRES, P. et ZANCARINI-FOURNEL, M. (dir.). 68, Une Histoire collective [1962-1981]. Paris : Éditions la Découverte. p. 285-290.

LOYER, E. (2008). Mai 68 dans le texte. Paris : Complexe.

MERLE, R. (2008). Derrière la vitre. Paris : Gallimard, « Folio ».

ROSANVAllon, P. (2018). Notre Histoire intellectuelle et politique 1968-2018. Paris, Seuil.

WEBER, H. (1998). Que reste-t-il de mai 68 ? Essai sur les interprétations des événements ». Paris, Seuil, « Points ». 


\section{NOTES}

1. Cité par Patrick Combes $(1968,160)$ de Les Parleuses (Minuit, 1974). Entretien avec X. Gautier.

2. Cité dans le tome III de la Correspondance de Flaubert, app. II. p. 882.

3. Livre qui rassemble également deux autres romans de Merle: Week-end à Zuydcoote et La Mort est mon métier.

4. Sotenville est un personnage de Georges Dandin de Molière). Il existe bien des Sotteville en France, dans l'Eure et dans la Manche.

\section{RÉSUMÉS}

À l'instar de Patrick Combes dans son ouvrage de 1984 La Littérature et le mouvement de mai 68, nous nous interrogerons sur les romans dont le thème central ou l'arrière-fond historique est la révolte survenue il y a 50 ans en France.

Ainsi, nous nous intéresserons plus particulièrement à trois romans publiés quelques année après les événements : Derrière la vitre (1970), de Robert Merle, Les deux printemps(1971), de Raymond Jean et L'Irrévolution (1971), de Pascal Lainé, dont les personnages et/ou les narrateurs sont des enseignants, ce qui fait d'eux des acteurs ou de simples témoins au plus près des préoccupations et des inquiétudes de la jeunesse étudiante qui voient surgir la mise en cause de la société et de l'école dans les années soixante du siècle dernier.

Like Patrick Combes in his book of 1984, La Littérature et le mouvement de mai 68, we willquestion the novels whose central theme or the historical background is the revolt that happened in may 68, in France.

We will be particulary interested in three novels published a few years after the events: Derrière la vitre (1970) of Robert Merle; Les deux printemps (1971) of Raymond Jean and L'Irrévolution (1971), of Pascal Lainé whose characters and/or narrators are teachers, which make them actors or mere witness close to youth student concerns and the questioning of society and the school int the sixties of last century.

\section{INDEX}

Mots-clés : Mai 68, littérature française du XXème siècle, Jean (Raymond), Lainé (Pascal), Merle (Robert)

Keywords : May 68, French literature of the 20th century, Jean (Raymond), Lainé (Pascal), Merle (Robert)

\section{AUTEUR}

\section{LUÍS CARLOS PIMENTA GONÇALVES}

Universidade Aberta / IELT (FCSH-NOVA)

Luis.Goncalves[at]@uab.pt 